\title{
MARRELlA and the Verification of an Embedded System
}

\author{
Dominique Ambroise $^{1}$, Patrick Augé ${ }^{1}$, Kamel Bouchefra ${ }^{2}$, and Brigitte Rozoy ${ }^{1}$ \\ 1 Laboratoire de Recherche en Informatique, \\ Université Paris 11, Bât. 490, 91405 Orsay Cedex France \\ \{ambroise, auge, rozoy\}@lri.fr \\ 2 Laboratoire d'Informatique de Paris Nord, Université Paris 13, \\ Avenue Jean-Baptiste Clément, 93430 Villetaneuse, France \\ kb@lipn.univ-paris13.fr
}

\begin{abstract}
We present the architecture of MARRELLA, a tool designed for simulation and verification of distributed systems. The input of the tool is an event driven language whereas the underlying model is event structures. It gives the possibilities of generating one, some or all the executions of any distributed program. We have tested the tool for the verification of an embedded system. The corresponding results are reported here as well as those obtained with equivalent tools applied to the same case study. These experimental results show then the efficiency of MARRELLA.
\end{abstract}

\section{Introduction}

It is now well known and recognized that one execution of a distributed program may be symbolized by a partial order. In the same spirit, prime event structures can be used to exhibit in a single object all possible executions. Thus, their use may become of a great help for formal specifications, simulations and verifications of such programs. Inherently, our method is based on these prime event structures and exploits techniques that unfold the behaviors of programs into acyclic transition systems, the nodes of which are global states. Then we exploit the properties of the structure to reduce the graph.

Another version of this idea has already been used for verification purposes : unfolding of occurrence Petri nets are constructed. Numerous other techniques have also been used for reducing the size of the graph and for on the fly verification [Esp. Röm. 99]. In case of distributed programs and as it partially avoids the famous state explosion problem, this model is well adapted for efficient simulations, even exhaustive, thus for verifications. Substantial efficiencies are obtained as the enumeration of all possible interleaving is avoided.

\section{MARRELla}

The tool may be viewed either as a simulator or as a model checker: starting with a concrete distributed algorithm, it efficiently constructs the associated graph of states. It is theoretically based on prime event structures with binary conflicts. 
The prime event structure $S$ constitutes the abstract level, not necessarily implemented but used to derive properties of the graph. Whereas a partial order representation stands for one execution of a distributed program, an event structure exhibits in a single object all its possible executions. In that sense event structures are close to Petri Nets with which they have strong connections [Wins. Niel. 95], while prime event structures are a special case related to traces [Maz. 87]. This set of configurations satisfies certain important domains properties not far from those of lattices : it may be called a budding lattice. Roughly speaking, its regularity allows efficient traversal algorithms.

First, the input is a distributive reactive asynchronous algorithm written in an Estelle or in a SDL like language [Est. 88, SDL 88]. Then MARrella implements an object, some kind of network the nodes of which are automaton equipped with buffers and communicating by channels [Amb. Roz. 96]. It allows constructing $G(R)$, the unfolded-labeled graph of states of the system, actually implemented totally or piece by piece in case it is too big.

This later graph is shown to be isomorphic to the graph of configurations of a prime event structure : $G(R) \approx \operatorname{Conf}(S)$. Therefore, using this result, efficient constructions and traversals of this graph may be implemented. Latter on, depending on the properties to be shown, this graph is folded into a smaller equivalent one.

\section{Construction and Observation Strategies}

As it benefits by the steady structure of the budding lattice, MARRELLA uses strategies to represent, reduce and examine the graph in an efficient way. First, the collection of events generated by processes is constructed on the fly, together with their dependence relation (a partial order) and their conflict relation (a binary irreflexive relation). Then two strategies are possible, depending on the properties to be tested.

Instead of the classical interleaving construction, that is heavy in space and time, we use an economical strategy that constructs a tree that covers the graph and is linear in the number of states. Here every state is constructed, thus the gain is not in the number of states, but in the number of comparisons : using an enumeration that labels the event on the fly, the algorithm "knows" which state has already been constructed and does not try to re-construct an already existent one. It is a breadth first construction with the following shape :

- Constructing the states of depth $(\rho+1)$ starting with those of depth $(\rho)$.

- Initialize $\mathrm{S}(\rho+1)$ at $\phi$.

for all state $\mathrm{s}$ in $\mathrm{S}(\rho)$ do

for all event e allowed in s do

if label $(\mathrm{s}) \ll \mathrm{e}$ then

fi

add $\mathrm{s}+\mathrm{e}$ to $\mathrm{S}(\rho+1)$
label $(\mathrm{s}+\mathrm{e}) \longleftarrow \mathrm{e}$

od

od 
The full version can be found in [Amb. Roz. 96]. The figure 1.a gives an example of such graph construction. A second strategy, called CoMAx, uses concurrent steps and constructs a tree too. It consists in maximizing the number of independent transitions that are executed in parallel. This tree has less states than a covering tree, and is such that each reachable state of the original graph is covered by at least one state of the constructed tree : thus detection of deadlocks or stable properties is available. It is not already published, but a preliminary version can be found in (http://lri.fr/ ambroise/marrella.html). Its main new idea is that, at each step, several independent processes may act together. Then a careful investigation of the conflict relation allows to backtrack some actions when necessary. An example is given in Fig. 1.b.
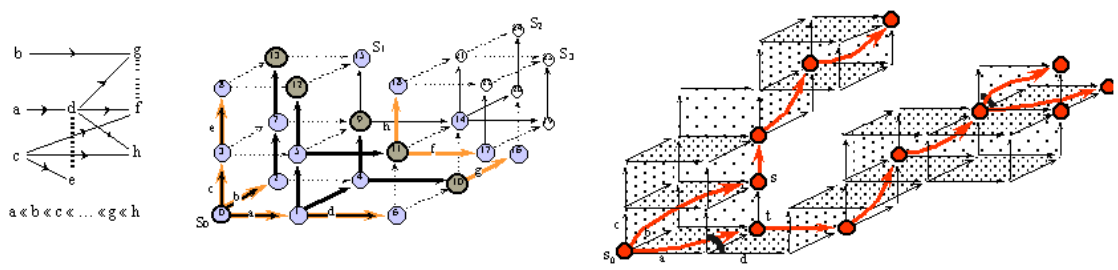

Fig. 1. (a) Atomic construction

Fig. 1. (b) Traversing of the graph

\section{Experiment with MARRELla on an Embedded System}

Collaboration has been initiated by electronic researchers and computer scientists. The goal was to re-write and verify the AsTRE architecture [Bou. 97], an agent based embedded system for avoiding road collision risks. This system processes data acquisition issued from the road traffic scenery, constructs a model of the environment and outputs diagnostics on collisions risks. Together, we have specified new architectures, focussing on the control scheme. The first one is a rather centralized control, corresponding to architecture with hard constraints. The second one is distributed, with relaxed constraints.

These new specifications of the AsTRE architecture have been verified with MARRELla as well as with other tools: OBJECTGEODE, a standard commercial tool, CADP, that is based on bisimulation, SPIN, based on static partial order reductions and SPIN ULG, based on dynamic partial order reductions. We give here the results and compare them. To make pertinent comparisons, both architectures have been first described with the SDL description language. Then translators, based on IF, are used to obtain the descriptions in the language supported by each tool. The following table reports the results when using respectively ObjectGeode, Marrella, Cadp, Spin and Spin Ulg. We compare here the number of states generated by each tool in the same conditions. We give them here for the two architectures and various data flows. 
Table 1. Simulation results, number of states constructed by each tool

\begin{tabular}{lrrrrrr}
\hline & \multicolumn{3}{c}{ Architecture 1 } & \multicolumn{3}{c}{ Architecture 2 } \\
\hline Tools & Flow 1 & Flow 2 & Flow 3 & Flow 1 & Flow 2 & Flow 3 \\
\hline OBJECTGEODE & 2028 & 1530 & 4321 & 19372 & 475285 & 4000000 \\
CADP & 1579 & 1448 & 2618 & 7543 & 59486 & 325195 \\
SPIN & 215 & 274 & 483 & 4315 & 75828 & 1034899 \\
SPIN UlG & 85 & 169 & 375 & 1388 & 48699 & 636218 \\
MARRELLA & 105 & 78 & 152 & 81 & 29486 & 137863 \\
\hline
\end{tabular}

Thus, considering the number of states, MARRELla (more or less together with SPIN Ulg and CADP) is clearly among the best, the reduction ratio going from 3 to 40 .

\section{Conclusion}

Although being only a toy written with a LisP like language, MARRELLA has been carefully implemented, thus spares memory. Moreover, precise and neat algorithms benefit from the trace properties of prime event structures and thus gain in avoiding the enumeration of equivalent interleaving.

Tested comparatively with other known tools, MARRELlA has proved its efficiency. The question now is to evaluate whether it is easy to use for a nonspecialist !

\section{References}

[Amb. Roz. 96] Ambroise, D., Rozoy, B. Marrella : a tool to analyse the graph of states. In: Parallel Processing Letters. Vol 6, $\mathrm{n}^{\circ} 4,583-594$. (1996)

[Bou. 97] K. Bouchefra, Formal model for road traffic collision risk avoidance. In: IFAC-IFIP Conference on Control of Industrial Systems (Crujic, Borne, Ferney, Ed.). Elsevier Science Ltd. Belfort, France (1997).

[Esp. Röm. 99] J. Esparza, S. Römer, An unfolding algorithm for synchronous product of transition systems, In: Proceedings of the $12^{\text {th }}$ International Conference on Concurrency Theory, Concur'99, Eindhoven, The Netherlands, August 1999, LNCS. $n^{\circ} 1664,2-20,1999$

[EST. 88] ESTELLE: A Formal Description Based on an Extended State Transition Model. ISO-9074, Geneva (1988)

[Maz. 87] A. Mazurkiewicz, Trace Theory, Advanced Course on Petri Nets, In: Bad Honnef, Germany LNCS $n^{\circ} 254$, 269-324.

[SDL 88] SDL : Recommendation Z. 100, Specification and Description Language. ITU-T, Geneva (1988)

[Wins. Niel. 95] G. Winskel, M. Nielsen, Models for concurrency, In: Handbook of Logic in Computer Science (S. Abramsky, DM. Gabbay, TSE. Maibaum eds.). 